\title{
Current status of robotic training in the UK - a trainees perspective
}

\author{
Katie E Chan ${ }^{1}$, Nikhil Vasdev ${ }^{2}$ \\ ${ }^{I}$ Department of urology Royal Devon and Exeter Hospital, UK. \\ ${ }^{2}$ Hertfordshire and South Bedfordshire Urological Robotic Cancer Centre, Department of urology, Lister Hospital, UK.
}

Received September 21, 2013; Revised December 23, 2013; Accepted December 26, 2013; Published Online December 29,2013

\section{Educational Article}

\begin{abstract}
The validation of robotic surgery in a growing number of operative procedures has increased its acceptance nationwide and its usage is becoming widespread. Training needs to reflect this fast paced environment to ensure that surgeons continue to progress competently and safely. Current surgical training in the UK is validated through the Intercollegiate Surgical Curriculum Programme (ISCP) with progression assessed through an Annual Review of Curriculum Progress (ARCP). There has been some resistance to this since its introduction and many trainees remain dissatisfied with this programme. Training in robotic surgery is currently focused through fellowships with little regular exposure to urology trainees at more junior levels. Robotic simulation provides a useful adjunct to training for both technical and non-technical skills. Its usage is particularly valuable to more inexperienced trainees but may be of limited benefit in those with more advanced skills. Training programmes such as the fundamental skills in robotic surgery (FSRS) have been created to facilitate robotic training and it is likely that the future of robotic surgery training will include a combination of theoretical learning, training programmes and fellowship training.
\end{abstract}

Keywords: Training; Robotic; Simulation; Programme; Fellowship; Validation

\section{Introduction}

Robotic surgery has increased in popularity since its introduction in 2003. Its validation in a growing number of operative procedures has increased its acceptance nationwide and its usage is becoming widespread. Initial training of robotic surgeons relied heavily on industry and it is this training which has helped to guide the first wave of robotic urological surgeons through their initial learning curve. The rapid introduction of robotic surgery throughout urology necessitates the introduction of standardized training methods.

\section{Background}

At present surgical training programmes vary greatly. The traditional approach to surgical training was an apprentice-

Corresponding author: Nikhil Vasdev FRCS (Urol); Royal College of Surgeons of England / British Association of Urological Surgeons National Robotic Urological Fellow, Department of Urology, Hertfordshire and South Bedfordshire Urological Robotic Cancer Centre, Lister Hospital, UK.

Email: nikhilvasdev@doctors.org.uk

Cite this article as:

Chan KE, Vasdev N. Current status of robotic training in the UK -

a trainees perspective. Int J Cancer Ther Oncol 2014; 2(1):02013. DOI: $10.14319 /$ ijcto.0201.3 ship model with focus on passing the necessary membership examinations and completion of a pre-set number of years. In the UK a formalised surgical training programme was introduced in 2007 in response to the introduction of the European Working Time Directive and concerns that decreased working hours would compromise training and in turn patient care. This programme, the Intercollegiate Surgical Curriculum Programme (ISCP), focuses on competency based progression in academia, technical skills (operative and clinical) and non-technical skills (leadership, communication, situational awareness and decision making). Progress is reviewed on a regular basis through an Annual Review of Curriculum Progress (ARCP) panel at which development in these areas is assessed. Training assessment is formalised through the ISCP website.

This is an electronic platform where trainees request assessments in the above areas from consultants in the form of work based assessments (WBAs). This system is not without its flaws and its introduction was met with a degree of resistance. A survey of trainee opinions about ISCP in 2008 found a high degree of dissatisfaction across all areas. ${ }^{1,2} \mathrm{~A}$ repeat of this survey 3 years later has shown a significant 
improvement but overall, trainees remain dissatisfied by the ISCP. $^{3}$

Despite this the ISCP does provide a good platform for structured learning with regular assessment and feedback. It also sets the benchmark for what to achieve in that academic year. When used earnestly by trainees it can encourage development and progression.

\section{The importance of a training programme in urology}

Training needs to occur in order to progress the next generation of robotic surgeons however, it is important that knowledge and skills in robotic surgery can be gained whilst in a controlled environment without undue risks to patients. In addition to this there is increasing emphasis on validation of skills and competency. The need for a robotic surgical curriculum has been highlighted by established experts ${ }^{4-6}$ as well as trainees. Curriculum delivery through a stepwise approach has been suggested firstly with acquisition of knowledge and understanding of the basic steps involved in the surgical procedure. Next with practical skills under expert supervision and finally with independent practice ${ }^{7}$ (see Figure 1). Emphasis has been placed on the importance of structured competency based progression with a minimum level of proficiency defined prior to commencing independent practice.

\begin{tabular}{|ll} 
1. & Online (theoretical) training \\
2. & Simulation and observation \\
3. & Fellowship \\
4. & Certification \\
5. & Independent surgery
\end{tabular}

Fig .1: Outline of robotic training.

\section{Simulation training}

Specific robotic simulators have been designed for training in robotic surgery and there are 5 main simulators available; Robotic Surgical Simulator (RoSS), SimSurgery Educational Platform (SEP), ProMis, Mimic dV-Trainer, and the Da Vinci Skills Simulator. Other than RoSS, all these have been assessed for face, construct and content validity. ${ }^{1}$ There does not appear to be one simulator that is superior to any other in terms of educational benefit but comparison is difficult due to the lack of standardised assessment between simulators. ${ }^{1}$ Robotic simulation has been suggested as a tool for validating competency through the measurement of progression through the learning curve. ${ }^{8}$ Indeed the usage of robotic simulator training has been associated with a significant reduction in task completion time and economy of movement. ${ }^{9}$ In practice, it has also been shown to be cost effective ${ }^{10,11}$, realistic and easy to use. ${ }^{12}$ Novice surgeons appear to have the greatest benefit from simulation training with more experienced surgeons demonstrating minimal improvement only. ${ }^{13,14}$ This may be because at present simulation focuses on generic basic skills such as hand eye coordination and therefore simulation training, though useful in the preliminary stages of learning, may not confer additional benefit in advanced surgical skill. ${ }^{1,15}$

A more generalised non-robotic training programme was introduced to urology trainees in south London. This was found to be useful in testing both technical and non-technical skills. Feedback from trainees in this course was positive with $90 \%$ of participants keen to have more participation in simulation training. ${ }^{12}$

\section{Training programmes}

1. Lister Hospital, Stevenage (BAUS accredited)

2. UCLH, London

3. Guys Hospital, London

4. Royal Marsden Hospital, London

5. Addenbrookes Hospital, Cambridge

6. Bristol urological institute

Fig. 2: Robotic fellowship programmes.

Robotic training programmes which use a combination of tutorials, observation of live surgery and skills training with simulation have been described (see Figure 2). ${ }^{11,14,16,17}$

The Fundamental Skills in Robotic Surgery (FSRS) training programme was created using curriculum exercises from the Fundamentals of Laparoscopic Surgery (FLS) course. A short tutorial on the usage of the Da Vinci Surgical Simulator (DvSS) was followed by three main skills tests; suture pass, ball skills and 4th arm manipulation. Their study showed favorable results compared to controls in the advancement of ball pass skills but less so in suture skills and 4th arm manipulation.

Dulan et al also base their training programme on elements from the FLS course whilst also incorporating an online tutorial in robotic surgery from Intuitive Surgical. ${ }^{11}$ This was followed by 9 skills exercises practiced on the DvSS to cover 16 specific skills which were assessed using the FLS criteria. On course completion all participants had substantial improvements in scores. Content for their course was determined by a group of expert robotic surgeons, and this course has been assessed for context, face and construct validity. ${ }^{18-20}$

Buchs et $\mathrm{al}^{17}$ also present their course based training in basic generic robotic skills involving a 2 day course of theory, practical sessions in a dry lab and with cadavers and observation of live robotic surgery. Participants who had completed the course went on to practice robotic surgery in $46 \%$ of cases whereas only $6 \%$ did so pre-course. ${ }^{17}$

For more advanced training and education Rashid et al have presented their approach to robotic training in a fellowship setting beginning with Da Vinci certification followed by 
stepwise introduction of practical hands on experience in the steps of prostatectomy with progress measured using a 1-5 analogue scale. Each assessment was logged in a digitally maintained diary. This has been shown to be successful in introducing steps of surgery in a controlled way which aids progression of the trainee without leading to delays in operative time or endangering patient safety. They found that both their fellowship trainees improved in completion time with the number of procedures performed and were able to complete a prostatectomy in a timely fashion by the end of their training. ${ }^{16}$

Training of robotic surgeons began with industry lead courses and has since heavily relied on these as the benchmark for introduction to robotics. More recently there has been a call to move away from industry lead training and the FSRS course has been suggested as an alternative. ${ }^{5}$ The FSRS course and the training programme by Dulan et al appear to make the greatest impact on novice surgeons much like basic simulation training. For more advanced robotic trainees a programme such as that suggested by Rashid et al is a good way of maintaining training whilst ensuring patient safety.

The electronic monitoring of progression mirrors that of current training in the UK with the ISCP platform. Although this training program clearly has its benefits it is more time consuming to arrange and requires a trainer who is skilled in the assessment techniques used. For this type of assessment to be useful nationwide it would require inter-rater reliability as well as being feasible to arrange at specialist robotic training centres.

\section{Fellowship}

The majority of robotic surgical training is carried out as part of a fellowship programme. Currently in the UK there are 6 robotic fellowships with only one that is BAUS accredited (see Figure 3 ). These are extremely competitive receiving applications internationally as well as from within the UK. Interest in these centres also comes from established urological consultants who are wishing to extend their practice to incorporate robotic surgery.

\section{Intuitive robotic fellowship grant \\ 2. Ethicon biosurgery passport bursaries \\ 3. Chitra Sethia Centre Bursaries \\ 4. The Urology Foundation}

Fig. 3: Support for fellowship.

Robotic fellowships have the advantage of being based in well-established teaching hospitals with high volumes of robotic cases and robotic experience. Experience in a high volume centres has been associated with the subsequent formation of a safe robotic unit in terms of functional and oncological outcome. ${ }^{21}$
Despite the positive impact on individual training for robotic fellowships its introduction can be contentious. There is a risk that the presence of robotic fellows can impact negatively upon the training for established urology SPRs. Therefore there has to be careful consideration regarding the division of labour and the provisions of training for all urology trainees. The role of the urology SPR can also be threatened by the introduction of advanced surgical nurse practitioners as bed side assistants in robotic surgery.

Their introduction has added stability to the assistant role which may otherwise be a periodically rotating post with an adjustment phase required for each transition. Their relative wealth of experience may result in many urology trainees being outperformed when first learning to assist in robotics and therefore nursing assistants may become the favoured choice by many consultants. Conversely some may find the role of the advanced surgical nurse practitioner has a positive effect as the presence of a constant and capable bedside assistant may allow trainees to spend time learning directly at the console with the supervising surgeon or at a dual console where these are present.

\section{Funding and support}

There are several financial considerations when undertaking a career in robotic surgery. Courses are expensive and yet necessary for progression although a number of small grants and bursaries are available for support (see Figure 3 ). The Urology Foundation supports consultants and their teams to further their education in robotic surgery through preceptorship programmes. They have also founded a mentorship programme for centres keen to enrol a robotic surgical unit at their home institution as well as a two fellowship training opportunities. In addition to small grants and bursaries, some fellowships will come with some salary support in return to service provision either through an on call capacity or by doing sessions in clinic or small elective cases.

\section{Assessment of training}

Assessment of robotic trainees is essential to ensure competency based progression prior to independent practice. For this to be useful it must be valid, reliable, feasible and have education impact. ${ }^{22}$ Part of the challenge of creating adequate assessment is defining the level of competency required. Methods of assessment can be categorized as per Figure 4.

1. Educational work based assessment

2. Logbook assessment

3. Annual review of progress

4. Examinations

5. Successful completion of training modules/courses

Fig. 4: Training assessment. 
This is simpler in testing knowledge which can be measured through examinations. However assessment of competence in technical and non-technical skills is more challenging. Currently assessment of both of these occurs through the completion of WBAs and these have been used widely in the ISCP training programme.

Between regions and trainees the assessment system must be standardised so that trainees all reach the same competency skills expected at each level. This can be difficult to achieve on a multinational basis with high numbers of assessors as each of these will need individual training in methods of education and assessment.

\section{Conclusions}

Urology is ever evolving in its approaches to oncological and benign conditions. There is increasing focus on minimally invasive and in particular robotic surgery. Training needs to reflect this fast paced environment to ensure that surgeons continue to progress competently and safely. A formal training programme in robotic surgery has been suggested to accomplish this however there are several challenges in its introduction specially with the introduction of complex robotic urological procedures such as robotic cystectomy and intracorporeal reconstruction techniques. ${ }^{23}$ The suggested format includes a pre-clinical or theoretical phase followed by simulation training whilst progressing to operative console training and eventually independent practice. Provision of pre-clinical training may be through lectures either on a local basis or through distance learning. Clinical experience is usually through fellowship training, however as robotic surgery becomes more established it is sensible to assume that this may occur at an increasingly more junior level. Simulation training has been suggested to complement clinical training by creating a safe environment in which refinement in technical and non-technical skills can occur. Specific multimodality training programmes have been designed and tested though an ideal cost effective, feasible, valid and educationally positive training programme is still awaited.

Current assessment methods available for trainees in the UK have been through work based assessments as part of the ISCP. There has been some resistance to this since its introduction but this has improved in the years since, perhaps suggesting that its usage has become more accepted amongst trainees. The ISCP could be used for assessment in robotic surgery. This would have the advantage of standardising assessment but its usage may not be popular in trainees from outside the UK who are not familiar with this training assessment modality. A more refined version of the ISCP may be an alternative, taking into account the negative comments from previous trainees and improving upon this.
For trainees there are lots of elements to consider when contemplating a career in robotic surgery. Training is longer and fellowships are competitive and may require financial support through grants or bursaries. Though this may put off some trainees there is an attraction in being close to the forefront of urological surgery and robotic surgery remains a highly competitive subspecialty.

The implementation of a robotic surgery training programme is an exciting opportunity to create a standardised educational platform to train the urological surgeons of the future, thus moving robotic surgery to the forefront of creating a safe and proficient workforce. Appropriate consideration of the controversies and challenges involved will smooth its introduction and make it an attractive option for urology trainees.

\section{Conflict of interest}

The authors declare that they have no conflicts of interest. The authors alone are responsible for the content and writing of the paper.

\section{References}

1. Abboudi H, Khan MS, Aboumarzouk O, Guru KA, Challacombe B, Dasgupta P, Ahmed K. Current status of validation for robotic surgery simulators a systematic review. BJU Int 2013; 111:194-205.

2. Pereira EA, Dean BJ. British surgeons' experiences of mandatory online workplace-based assessment. J R Soc Med 2009; 102:287-93.

3. Pereira EA, Dean BJ. British surgeons' experiences of a mandatory online workplace based assessment portfolio resurveyed three years on. J Surg Educ 2013; 70:59-67.

4. Lee JY, Mucksavage P, Sundaram CP, McDougall EM. Best practices for robotic surgery training and credentialing. J Urol 2011; 185:1191-7.

5. Buffi N, Van Der Poel H, Guazzoni G, Mottrie A; Junior European Association of Urology (EAU) Robotic Urology Section with the collaboration of the EAU Young Academic Urologists Robotic Section. Methods and priorities of robotic surgery training program. Methods and Priorities of Robotic Surgery Training Program. Eur Urol 2014; 65:1-2.

6. Ficarra V, Wiklund PN, Rochat CH, Dasgupta P, Challacombe BJ, Sooriakumaran P, Siemer S, Suardi N, Novara G, Mottrie A. The European Association of Urology Robotic Urology Section (ERUS) survey of robot-assisted radical prostatectomy (RARP). BJU Int 2013; 111:596-603. 
7. Sachdeva AK. Acquiring skills in new procedures and technology: the challenge and the opportunity. Arch Surg 2005; 140:387-9.

8. Khan N, Abboudi H, Khan MS, Dasgupta P, Ahmed $\mathrm{K}$. Measuring the surgical 'learning curve': methods, variables and competency. BJU Int 2013.

9. Tergas AI, Sheth SB, Green IC, Giuntoli RL 2nd, Winder AD, Fader AN. A pilot study of surgical training using a vir-tual robotic surgery simulator. Jsls 2013; 17:219-26.

10. Rehman S, Raza SJ, Stegemann AP, Zeeck K, Din $\mathrm{R}$, Llewellyn A, et al. Simulation-based robot-assisted surgical train-ing: A health economic evaluation. Int J Surg 2013; 11:841-6.

11. Dulan G, Rege RV, Hogg DC, Gilberg-Fisher KM, Arain NA, Tesfay ST, Scott DJ. Developing a comprehensive, profi-ciency-based training program for robotic surgery. Surgery 2012; 152:477-88.

12. Shamim Khan M, Ahmed K, Gavazzi A, Gohil R, Thomas L, Poulsen J, et al. Development and implementation of cen-tralized simulation training: evaluation of feasibility, acceptability and construct validity. BJU Int 2013; 111:518-23.

13. Davis JW, Kamat A, Munsell M, Pettaway C, Pisters L, Matin S. Initial experience of teaching robot-assisted radical prostatectomy to surgeons-in-training: can training be evaluated and standardized? BJU Int 2010; 105:1148-54.

14. Stegemann AP, Ahmed K, Syed JR, Rehman S, Ghani K, Autorino R, et al. Fundamental skills of robotic surgery: a multi-institutional randomized controlled trial for validation of a simulation-based curriculum. Urology 2013; 81:767-74.

15. Hung AJ, Jayaratna IS, Teruya K, Desai MM, Gill IS, Goh AC. Comparative assessment of three standardized robotic surgery training methods. BJU Int 2013; 112:864-71.

16. Rashid HH, Leung YY, Rashid MJ, Oleyourryk G, Valvo JR, Eichel L. Robotic surgical education: a systematic approach to training urology residents to perform robotic-assisted laparoscopic radical prostatectomy. Urology 2006; 68:75-9.

17. Buchs NC, Pugin F, Volonte F, Hagen ME, Morel $P$. Impact of robotic general surgery course on participants' surgical practice. Surg Endosc 2013; 27:1968-72.

18. Dulan G, Rege RV, Hogg DC, Gilberg-Fisher KM, Arain NA, Tesfay ST, Scott DJ. Proficiency-based training for ro-botic surgery: construct validity, workload, and expert levels for nine inanimate exercises. Surg Endosc 2012; 26:1516-21.

19. Dulan G, Rege RV, Hogg DC, Gilberg-Fisher KK, Tesfay ST, Scott DJ. Content and face validity of a comprehensive robotic skills training program for general surgery, urology, and gynecology. Am J Surg 2012; 203:535-9.
20. Arain NA, Dulan G, Hogg DC, Rege RV, Powers $\mathrm{CE}$, et al. Comprehensive proficiency-based inanimate training for robotic surgery: reliability, feasibility, and educational benefit. Surg Endosc 2012; 26:2740-5.

21. Lumen N, Van Praet C, De Troyer B, Fonteyne V, Oosterlinck W, Decaestecker K, Mottrie A. Safe introduction of robot-assisted radical prostatectomy after a training program in a high-volume robotic centre. Urol Int 2013; 91:145-52.

22. Beard JD. Assessment of surgical skills of trainees in the UK. Ann R Coll Surg Engl 2008; 90:282-5.

23. Vasdev N, Lamb B, Lane T, Boustead G, Adshead J. Robotic Cystectomy : Important considerations before commencing the procedure independently. Int J Cancer Ther Oncol 2013; 1:01017. 